\title{
Algebras of quotients with bounded evaluation of a normed semiprime algebra
}

\author{
by \\ M. Cabrera (Granada) and Amir A. Mohammed (Mosul)
}

\begin{abstract}
We deal with the algebras consisting of the quotients that produce bounded evaluation on suitable ideals of the multiplication algebra of a normed semiprime algebra $A$. These algebras of quotients, which contain $A$, are subalgebras of the bounded algebras of quotients of $A$, and they have an algebra seminorm for which the relevant inclusions are continuous. We compute these algebras of quotients for some norm ideals on a Hilbert space $H: 1$ ) the algebras of quotients with bounded evaluation of the ideal of all compact operators on $H$ are equal to the Banach algebra of all bounded linear operators on $H, 2$ ) the algebras of quotients with bounded evaluation of the Schatten $p$-ideal on $H$ (for $1 \leq p<\infty$ ) are equal to the Schatten $p$-ideal on $H$. We also prove that the algebras of quotients with bounded evaluation on the class of totally prime algebras have an analytic behavior similar to the one known for the bounded algebras of quotients on the class of ultraprime algebras.
\end{abstract}

Introduction and preliminaries. Throughout this paper all algebras considered are associative over the field $\mathbb{K}$ equal to $\mathbb{R}$ or $\mathbb{C}$.

The notion of rings of quotients (in which two-sided ideals are used) was introduced by W. S. Martindale for prime rings in [5] and extended to semiprime rings by S. A. Amitsur in [1]. It is usual to define these rings of quotients using partially defined centralizers on essential ideals. However, for our purposes we prefer to give a somewhat more abstract presentation (see for example [9] or [2]). Given a semiprime algebra $A$, the right algebra of quotients of $A$, denoted here by $Q^{r}(A)$, is defined as the maximal algebra extension $Q$ of $A$ which satisfies the following conditions:

(i) if $q \in Q$, then there exists an essential ideal $I$ of $A$ with $q I \subseteq A$,

(ii) if $q \in Q, I$ is an essential ideal of $A$, and $q I=0$, then $q=0$.

Given $q$ in $Q^{r}(A)$, and an essential ideal $I$ of $A$ such that $q I \subseteq A$, the symbol $L_{q}^{I}$ will denote the mapping from $I$ into $A$ given by $L_{q}^{I}(x)=q x$ for

2000 Mathematics Subject Classification: Primary 46H20; Secondary 16S90, 46H10, $47 \mathrm{~B} 10$.

The first author is supported by Junta de Andalucía Grant FQM290. 
all $x$ in $I$. Following [6; Chapter 2], when $A$ is additionally a normed algebra, the right bounded algebra of quotients of $A$ is defined as the subalgebra of $Q^{r}(A)$ given by

$$
\begin{array}{r}
Q_{b}^{r}(A)=\left\{q \in Q^{r}(A): \text { there exists an essential ideal } I \text { of } A\right. \\
\text { such that } \left.q I \subseteq A \text { and } L_{q}^{I} \text { is bounded }\right\}
\end{array}
$$

endowed with the algebra seminorm

$$
\begin{aligned}
& \|q\|_{r}=\inf \left\{\left\|L_{q}^{I}\right\|: I \text { is an essential ideal of } A\right. \\
& \text { such that } \left.q I \subseteq A \text { and } L_{q}^{I} \text { is bounded }\right\} .
\end{aligned}
$$

It is clear that the inclusion of $A$ into $Q^{r}(A)$ becomes a continuous embedding of $A$ into $Q_{b}^{r}(A)$. In a similar fashion, using $Q^{l}(A)$ (the left algebra of quotients of $A$ ), one defines the left bounded algebra of quotients $\left(Q_{b}^{l}(A),\|\cdot\|_{l}\right)$. Also, the fact that the symmetric algebra of quotients of $A$ can be viewed as $Q^{s}(A)=Q^{r}(A) \cap Q^{l}(A)$ suggests defining the symmetric bounded algebra of quotients $\left(Q_{b}^{s}(A),\|\cdot\|_{s}\right)$ by

$$
Q_{b}^{s}(A)=Q_{b}^{r}(A) \cap Q_{b}^{l}(A) \text { and }\|\cdot\|_{s}=\max \left\{\|\cdot\|_{r},\|\cdot\|_{l}\right\} .
$$

Bounded algebras of quotients behave well on an interesting class of normed algebras first discussed by M. Mathieu in [6]. Recall that a normed algebra $A$ is ultraprime if there exists a positive number $K$ such that $K\|a\|\|b\| \leq\left\|M_{a, b}\right\|$ for all $a, b$ in $A$, where $M_{a, b}: A \rightarrow A$ is the two-sided multiplication operator defined by $M_{a, b}(x)=a x b$ for all $x$ in $A$. Bounded algebras of quotients provide analytic algebras of quotients on the class of ultraprime algebras in the following sense: if $A$ is an ultraprime algebra, then $Q_{b}^{r}(A)$ is an ultraprime algebra, and the inclusion of $A$ into $Q_{b}^{r}(A)$ is topological. Moreover, if $Q$ is a normed algebra such that $Q$ is a subalgebra of $Q^{r}(A)$ containing $A$, and the inclusion of $A$ into $Q$ is topological, then $Q$ is continuously embedded in $Q_{b}^{r}(A)$ (see [7; Theorem 4.1] and [8; Proposition 2.8]).

In Section 1, we will introduce the algebras of quotients with bounded evaluation for a normed semiprime algebra. Our construction involves the multiplication algebra and our treatment follows, as far as possible, the aforementioned construction of bounded algebras of quotients. Recall that the multiplication algebra $M(A)$ of an algebra $A$ is defined as the subalgebra of $L(A)$ (the algebra of all linear operators on $A$ ) generated by the identity operator $\operatorname{Id}_{A}$ and the set $\left\{L_{a}, R_{a}: a \in A\right\}$, where $L_{a}$ and $R_{a}$ are the operators of left and right (respectively) multiplication by $a$ on $A$. The starting point of our construction is a result which is purely algebraic in nature and which asserts that if $A$ is a semiprime algebra, then $M(A)$ is canonically embedded in $M\left(Q^{r}(A)\right)$. Using this result, for each $q$ in $Q^{r}(A)$ and each essential ideal $I$ of $A$ such that $q I \subseteq A$, we will consider the ideal $I^{r}$ 
of $M(A)$ generated by the set $\left\{R_{x}: x \in I\right\}$ and the $A$-valued mapping $E_{q}^{I^{r}}$ determined by the evaluation on $q$ of the elements of $I^{r}$. The right algebra of quotients with bounded evaluation $Q_{b e}^{r}(A)$ for a normed semiprime algebra $A$ arises when the operators $L_{q}^{I}$ in the definition of $Q_{b}^{r}(A)$ are replaced by the evaluation operators $E_{q}^{I^{r}}$.

Section 2 is devoted to the study of the algebras of quotients with bounded evaluation of some norm ideals on a Hilbert space $H$, namely the ideal $K L(H)$ of all compact operators and the Schatten $p$-ideals $\mathcal{C}_{p}(H)$ $(1 \leq p<\infty)$. We will begin by determining the bounded algebras of quotients for arbitrary norm ideals: if $A$ is a norm ideal on $H$, then the bounded algebras of quotients of $A$ are equal to $B L(H)$ (the Banach algebra of all bounded linear operators on $H)$. Our main results assert that $\mathcal{C}_{p}(H)(1 \leq p$ $<\infty)$ is closed under passing to algebras of quotients with bounded evaluation, while the algebras of quotients with bounded evaluation of $K L(H)$ are equal to $B L(H)$.

In Section 3 we will study the behavior of the algebras of quotients with bounded evaluation on the class of totally prime algebras introduced in [4]. Algebras of quotients with bounded evaluation provide analytic algebras of quotients for this class. So, for example, for the symmetric algebra, we will prove that: if $A$ is a totally prime algebra, then $Q_{b e}^{s}(A)$ is a totally prime algebra, and the inclusions of $A$ into $Q_{b e}^{s}(A)$ and of $M(A)$ into $M\left(Q_{b e}^{s}(A)\right)$ are topological. Moreover, if $Q$ is a normed algebra such that $Q$ is a subalgebra of $Q^{s}(A)$ containing $A$ and the inclusions of $A$ into $Q$ and of $M(A)$ into $M(Q)$ are topological, then $Q$ and $M(Q)$ are continuously embedded in $Q_{b e}^{s}(A)$ and $M\left(Q_{b e}^{s}(A)\right)$ respectively. An analogous result is also obtained for totally multiplicatively prime algebras, which were recently introduced by the authors in [3].

\section{Algebras of quotients with bounded evaluation of a normed} semiprime algebra. For our construction we shall begin with a general result on multiplication algebras that relies on the theory of generalized polynomial identities (see [2]). Let $A$ be a semiprime algebra and let $C$ denote the extended centroid of $A$ (that is, the centre of $Q^{r}(A)$ ). Let $\mathbf{X}$ be a countably infinite set (of "formal variables"); denote by $C\langle\mathbf{X}\rangle$ the free algebra over $C$ on $\mathbf{X}$, and by $Q^{r}(A)_{C}\langle\mathbf{X}\rangle$ the coproduct of the $C$-algebras $Q^{r}(A)$ and $C\langle\mathbf{X}\rangle$. The elements of $Q^{r}(A)_{C}\langle\mathbf{X}\rangle$ are generalized polynomial identities. A generalized polynomial identity $\phi$ is satisfied by $A$ if $s(\phi)=0$ for all (substitution) $C$-algebra homomorphisms $s: Q^{r}(A)_{C}\langle\mathbf{X}\rangle \rightarrow Q^{r}(A)$ such that $s(\mathbf{X}) \subseteq A$ and $s(q)=q$ for all $q$ in $Q^{r}(A)$.

Proposition 1. Let $A$ be a semiprime algebra and $Q, Q^{\prime}$ be subalgebras of $Q^{r}(A)$ such that $A \subseteq Q \subseteq Q^{\prime} \subseteq Q^{r}(A)$. Then for all $F$ in $M(Q)$ there 
exists a unique element $\widetilde{F}$ in $M\left(Q^{\prime}\right)$ such that

$$
\widetilde{F}(a)=F(a) \quad \text { for all a in } A,
$$

and the mapping $F \mapsto \widetilde{F}$ becomes an algebra monomorphism from $M(Q)$ into $M\left(Q^{\prime}\right)$.

Proof. Let $Q^{1}$ denote the unital envelope of $Q$ in $Q^{r}(A)$, that is, the subalgebra of $Q^{r}(A)$ generated by $Q$ and the unit 1 of $Q^{r}(A)$. For $p, q$ in $Q^{1}$, denote by $M_{p, q}^{Q}$ the multiplication operator on $Q$ defined by $M_{p, q}^{Q}(r)=p r q$ for all $r$ in $Q$. It is easy to see that

$$
M(Q)=\left\{\sum_{i=1}^{n} M_{p_{i}, q_{i}}^{Q}: n \in \mathbb{N}, p_{i}, q_{i} \in Q^{1}(1 \leq i \leq n)\right\} .
$$

We shall use the same notation for $Q^{\prime}$. For $F$ in $M(Q)$, if we choose $n$ in $\mathbb{N}$ and $p_{i}, q_{i}$ in $Q^{1}(1 \leq i \leq n)$ such that $F=\sum_{i=1}^{n} M_{p_{i}, q_{i}}^{Q}$, then it is obvious that the element of $M\left(Q^{\prime}\right)$ given by $F^{\prime}:=\sum_{i=1}^{n} M_{p_{i}, q_{i}}^{Q^{\prime}}$ satisfies the following condition:

$$
F^{\prime}(q)=F(q) \text { for all } q \text { in } Q,
$$

therefore confirming the existence of a multiplication operator $\widetilde{F}$ which satisfies (1). To prove the uniqueness of $\widetilde{F}$ it suffices to show that for $T$ in $M\left(Q^{\prime}\right)$ the condition $T(A)=0$ implies $T=0$. Let $T$ be an element of $M\left(Q^{\prime}\right)$ such that $T(a)=0$ for all $a$ in $A$. If we choose $n$ in $\mathbb{N}$ and $p_{i}, q_{i}$ in $Q^{\prime 1}(1 \leq i \leq n)$ such that $T=\sum_{i=1}^{n} M_{p_{i}, q_{i}}^{Q^{\prime}}$, then $\phi(\mathbf{x})=\sum_{i=1}^{n} p_{i} \mathbf{x} q_{i}$ is a generalized polynomial identity satisfied by $A$. By [2; Proposition 6.3.13], $T=0$. Finally, it is a straightforward verification (taking (2) into account) that the mapping $F \mapsto \widetilde{F}$ is an algebra monomorphism from $M(Q)$ into $M\left(Q^{\prime}\right)$.

The above proposition allows us to conclude that if $A$ is a semiprime algebra and if $Q, Q^{\prime}$ are subalgebras of $Q^{r}(A)$ such that $A \subseteq Q \subseteq Q^{\prime} \subseteq$ $Q^{r}(A)$, then the evaluation at elements of $A$ determines the corresponding inclusions for the multiplication algebras:

$$
M(A) \subseteq M(Q) \subseteq M\left(Q^{\prime}\right) \subseteq M\left(Q^{r}(A)\right),
$$

a fact that we will use without further mention.

For an ideal $I$ of an algebra $A$ we will denote by $I^{r}$ the ideal of $M(A)$ generated by the set $\left\{R_{x}: x \in I\right\}$.

Lemma 1. Let $A$ be an algebra. If $I$ is an ideal of $A$, then $I^{r}$ coincides with the left and right ideals of $M(A)$ generated by the set $\left\{R_{x}: x \in I\right\}$. 
Moreover,

$$
I^{r}=\left\{\sum_{i=1}^{n} M_{a_{i}, x_{i}}: n \in \mathbb{N}, a_{i} \in A^{1}, x_{i} \in I(1 \leq i \leq n)\right\},
$$

where $A^{1}$ denotes the unital envelope of $A$ in $Q^{r}(A)$.

Proof. Let $I$ be an ideal of $A$ and let $\mathcal{P}=\left\{\sum_{i=1}^{n} M_{a_{i}, x_{i}}: n \in \mathbb{N}, a_{i} \in A^{1}\right.$, $\left.x_{i} \in I(1 \leq i \leq n)\right\}$. Since $M_{a, b} M_{c, d}=M_{a c, d b}$ for all $a, b, c, d$ in $A^{1}$, it follows that $\mathcal{P}$ is a two-sided ideal of $M(A)$. For $x$ in $I$ and $a$ in $A^{1}$, taking into account the equalities $R_{x}=M_{1, x}$ and $M_{a, x}=L_{a} R_{x}=R_{x} L_{a}$, it is clear that $\mathcal{P}$ contains the set $\left\{R_{x}: x \in I\right\}$ and that $\mathcal{P}$ is contained in the left and right ideals of $M(A)$ generated by $\left\{R_{x}: x \in I\right\}$. This concludes the proof.

Now we introduce the operators that support the framework of the right algebra of quotients with bounded evaluation of a normed semiprime algebra $A$. Given $q$ in $Q^{r}(A)$, it is clear that the set $D=\{F \in M(A): F(q) \in A\}$ is a left ideal of $M(A)$, and that the evaluation mapping $E_{q}$ defined by $E_{q}(F)=F(q)$ for all $F$ in $D$ is $A$-valued. If $I$ is an ideal of $A$ such that $q I \subseteq A$, then the set $\left\{R_{x}: x \in I\right\}$ is contained in $D$, hence $I^{r}$ is also contained in $D$ as a consequence of Lemma 1 . Thus, we may consider the restriction of $E_{q}$ to $I^{r}$, which will be denoted by $E_{q}^{I^{r}}$.

TheOREm 1. Let $A$ be a normed semiprime algebra. Then

$$
\begin{array}{r}
Q_{b e}^{r}(A)=\left\{q \in Q^{r}(A): \text { there exists an essential ideal } I \text { of } A\right. \\
\text { such that } \left.q I \subseteq A \text { and } E_{q}^{I^{r}} \text { is bounded }\right\}
\end{array}
$$

is a subalgebra of $Q^{r}(A)$, and $\mathbf{|} \cdot \mathbf{I}_{r}: Q_{b e}^{r}(A) \rightarrow \mathbb{R}$ defined by

$$
\mid q \mathbf{|}_{r}=\inf \left\{\left\|E_{q}^{I^{r}}\right\|: I \text { is an essential ideal of } A\right.
$$

such that $q I \subseteq A$ and $E_{q}^{I^{r}}$ is bounded $\}$

is an algebra seminorm. Moreover,

(i) $Q_{b e}^{r}(A)$ is a subalgebra of $Q_{b}^{r}(A)$ containing $A$, and these inclusions are continuous. Precisely, $|a|_{r} \leq\|a\|$ for all a in $A$, and $\|q\|_{r} \leq \mid q \mathbf{|}_{r}$ for all $q$ in $Q_{b e}^{r}(A)$.

(ii) The inclusion of $M(A)$ into $M\left(Q_{b e}^{r}(A)\right)$ is continuous. Precisely, $\mid F \mathbf{|}_{r} \leq\|F\|$ for all $F$ in $M(A)$.

Proof. It is easy to prove that $Q_{b e}^{r}(A)$ is a subspace of $Q^{r}(A)$ and $\mid \cdot \mathbf{I}_{r}$ is a seminorm on $Q_{b e}^{r}(A)$. Let $p, q$ be in $Q_{b e}^{r}(A)$, and consider essential ideals $I, J$ of $A$ such that $p I \subseteq A, q J \subseteq A$ and $E_{p}^{I^{r}}, E_{q}^{J^{r}}$ are bounded. Note that $J I$ is an essential ideal of $A$ such that $p q J I \subseteq A$ and $(J I)^{r} \subseteq I^{r} \cap J^{r}$. Moreover, the inclusion $(J I)^{r} R_{q} \subseteq I^{r}$ is a consequence of the above lemma and of the fact that $M_{a, y x} R_{q}=M_{a, q y x} \in I^{r}$ for all $a$ in $A^{1}, x$ in $I$, and $y$ 
in $J$. Now, for $F$ in $(J I)^{r}$ we can write

$$
E_{p q}^{(J I)^{r}}(F)=F(p q)=F R_{q}(p)=E_{p}^{I^{r}}\left(F R_{q}\right),
$$

and so

$$
\left\|E_{p q}^{(J I)^{r}}(F)\right\|=\left\|E_{p}^{I^{r}}\left(F R_{q}\right)\right\| \leq\left\|E_{p}^{I^{r}}\right\|\left\|F R_{q}\right\| .
$$

Moreover, for $a$ in $A$ we have

$$
F R_{q}(a)=F(a q)=F L_{a}(q)=E_{q}^{J^{r}}\left(F L_{a}\right),
$$

hence

$$
\left\|F R_{q}(a)\right\|=\left\|E_{q}^{J^{r}}\left(F L_{a}\right)\right\| \leq\left\|E_{q}^{J^{r}}\right\|\left\|F L_{a}\right\| \leq\left\|E_{q}^{J^{r}}\right\|\|F\|\|a\|,
$$

and so

$$
\left\|F R_{q}\right\| \leq\left\|E_{q}^{J^{r}}\right\|\|F\| .
$$

From (3) and (4) it follows that $E_{p q}^{(J I)^{r}}$ is bounded and

$$
\left\|E_{p q}^{(J I)^{r}}\right\| \leq\left\|E_{p}^{I^{r}}\right\|\left\|E_{q}^{J^{r}}\right\|,
$$

so $p q$ lies in $Q_{b e}^{r}(A)$ and $\mid p q \mathbf{|}_{r} \leq\left\|E_{p}^{I^{r}}\right\|\left\|E_{q}^{J^{r}}\right\|$. Hence $\left|p q \mathbf{|}_{r} \leq\right| p \mathbf{|}_{r} \mid q \mathbf{|}_{r}$.

(i) For $a$ in $A$ and $F$ in $A^{r}$ we have

$$
\left\|E_{a}^{A^{r}}(F)\right\|=\|F(a)\| \leq\|F\|\|a\|,
$$

therefore $E_{a}^{A^{r}}$ is bounded and $\left\|E_{a}^{A^{r}}\right\| \leq\|a\|$, and so $a$ lies in $Q_{b e}^{r}(A)$ and $|a|_{r} \leq\|a\|$. Let $q$ in $Q_{b e}^{r}(A)$, and suppose that $I$ is an essential ideal of $A$ such that $q I \subseteq A$ and $E_{q}^{I^{r}}$ is bounded. Then, for all $x$ in $I$,

$$
\left\|L_{q}^{I}(x)\right\|=\|q x\|=\left\|E_{q}^{I^{r}}\left(R_{x}\right)\right\| \leq\left\|E_{q}^{I^{r}}\right\|\|x\|,
$$

hence $L_{q}^{I}$ is bounded and $\left\|L_{q}^{I}\right\| \leq\left\|E_{q}^{I^{r}}\right\|$, therefore $q$ lies in $Q_{b}^{r}(A)$ and $\|q\|_{r} \leq \mid q \mathbf{|}_{r}$.

(ii) Fix $F$ in $M(A)$. If $q$ is in $Q_{b e}^{r}(A)$ and $I$ is an essential ideal of $A$ such that $q I \subseteq A$ and $E_{q}^{I^{r}}$ is bounded, then $F(q) I \subseteq I^{r} F(q) \subseteq I^{r}(q) \subseteq A$; therefore, for each $G$ in $I^{r}$, we can write $E_{q}^{I^{r}}(G F)=G F(q)=E_{F(q)}^{I^{r}}(G)$. From this it follows that $\left\|E_{F(q)}^{I^{r}}(G)\right\| \leq\left\|E_{q}^{I^{r}}\right\|\|G\|\|F\|$, hence $E_{F(q)}^{I^{r}}$ is bounded and $\left\|E_{F(q)}^{I^{r}}\right\| \leq\left\|E_{q}^{I^{r}}\right\|\|F\|$. As a consequence, $\mid F(q) \mathbf{I}_{r} \leq\left\|E_{q}^{I^{r}}\right\|\|F\|$ and, taking the infimum over $I$, we have $\left|F(q) \mathbf{|}_{r} \leq\right| q \mathbf{|}_{r}\|F\|$. This implies that $|F|_{r} \leq\|F\|$.

Definition 1. Let $A$ be a normed semiprime algebra. The seminormed algebra $\left(Q_{b e}^{r}(A), \mathbf{|} \cdot \mathbf{I}_{r}\right)$ described in the previous theorem will be called the right algebra of quotients with bounded evaluation of $A$. Similarly one defines the left algebra of quotients with bounded evaluation of $A$ through $Q^{l}(A)$ as follows: 


$$
\begin{array}{r}
Q_{b e}^{l}(A)=\left\{q \in Q^{l}(A): \text { there exists an essential ideal } I \text { of } A\right. \\
\text { such that } \left.I q \subseteq A \text { and } E_{q}^{I^{l}} \text { is bounded }\right\}
\end{array}
$$

and

$$
\mathbf{|} q \mathbf{|}_{l}=\inf \left\{\left\|E_{q}^{I^{l}}\right\|: I \text { is an essential ideal of } A\right.
$$

$$
\text { such that } \left.I q \subseteq A \text { and } E_{q}^{I^{l}} \text { is bounded }\right\} \text {, }
$$

where $E_{q}^{I^{l}}$ denotes the $A$-valued mapping determined by the evaluation on $q$ of the elements of $I^{l}$ (the ideal of $M(A)$ generated by the set $\left\{L_{x}: x \in I\right\}$ ). Also, the symmetric algebra of quotients with bounded evaluation of $A$ is defined as the seminormed algebra $\left(Q_{b e}^{s}(A), \mathbf{I} \cdot \mathbf{I}_{s}\right)$ given by

$$
Q_{b e}^{s}(A)=Q_{b e}^{r}(A) \cap Q_{b e}^{l}(A) \text { and } \mathbf{I} \cdot \mathbf{I}_{s}=\max \left\{\mathbf{I} \cdot \mathbf{I}_{r}, \mathbf{|} \cdot \mathbf{I}_{l}\right\} \text {. }
$$

\section{Algebras of quotients with bounded evaluation for Schatten} ideals and compact operators on a Hilbert space. Throughout this section, $H$ will denote a Hilbert space over $\mathbb{K}$ with inner product $\langle\cdot, \cdot\rangle, L(H)$ the algebra of all linear operators on $H$, and $\left(B L(H),\|\cdot\|_{\infty}\right)$ the Banach algebra of all bounded linear operators on $H$ with the operator norm. As usual, for $x, y$ in $H, x \otimes y$ will denote the rank-one operator on $H$ defined by $(x \otimes y)(z)=\langle z, y\rangle x$ for all $z$ in $H$. It is well known that the subset $F L(H)$ of $B L(H)$ consisting of all finite-rank operators can be expressed as follows:

$$
F L(H)=\left\{\sum_{i=1}^{n} x_{i} \otimes y_{i}: n \in \mathbb{N}, x_{i}, y_{i} \in H(1 \leq i \leq n)\right\} .
$$

Moreover, $F L(H)$ is the sum of all minimal left ideals of $B L(H)$ (the socle of $B L(H))$ and is a minimal ideal of $B L(H)$ contained in every ideal of $B L(H)$. Recall also that a norm ideal on $H$ is an ideal $A$ of $B L(H)$ endowed with a norm $\|\cdot\|$ satisfying the following conditions:

(i) $\|x \otimes y\|=\|x\|\|y\|$ for all $x, y$ in $H$ (cross-property),

(ii) $\|F T G\| \leq\|F\|_{\infty}\|T\|\|G\|_{\infty}$ for all $T$ in $A$ and $F, G$ in $B L(H)$.

From these conditions, for $T$ in $A$ and $x, y$ in $H$ with $\|x\|=\|y\|=1$, it follows that

$$
\|T(x)\|=\|T(x) \otimes y\|=\|T(x \otimes y)\| \leq\|T\|\|x \otimes y\|_{\infty}=\|T\| .
$$

Hence $\|T\|_{\infty} \leq\|T\|$, and taking (ii) into account once again, we conclude that $A$ is a normed algebra.

For a bounded linear operator $T$ on $H$, we will denote by $T^{*}$ the adjoint operator of $T$. Note that if $A$ is a subalgebra (resp. left ideal, right ideal, ideal) of $B L(H)$, then it is clear that

$$
A^{*}:=\left\{T^{*}: T \in A\right\}
$$

is a subalgebra (resp. right ideal, left ideal, ideal) of $B L(H)$ and $*: A \rightarrow A^{*}$ 
is an algebra anti-isomorphism. Moreover, if $\|\cdot\|$ is an algebra norm on $A$, then it is clear that $\|\cdot\|^{*}: A^{*} \rightarrow \mathbb{R}$, defined by $\|S\|^{*}:=\left\|S^{*}\right\|$ for all $S$ in $A^{*}$, is an algebra norm on $A^{*}$. The normed algebra $\left(A^{*},\|\cdot\|^{*}\right)$ will be denoted by $(A,\|\cdot\|)^{*}$. It is easy to see that if $(A,\|\cdot\|)$ is a norm ideal on $H$, then $(A,\|\cdot\|)^{*}$ is also a norm ideal on $H$.

Our first goal is to determine the bounded algebra of quotients of those normed algebras that are norm ideals.

Theorem 2. Let $(A,\|\cdot\|)$ be a norm ideal on $H$. Then

$$
\left(Q_{b}^{r}(A),\|\cdot\|_{r}\right)=\left(Q_{b}^{l}(A),\|\cdot\|_{l}\right)=\left(Q_{b}^{s}(A),\|\cdot\|_{s}\right)=\left(B L(H),\|\cdot\|_{\infty}\right) .
$$

Proof. Let $H^{c}$ denote the conjugate Hilbert space of $H$. Note that $\left(H, H^{c},\langle\cdot, \cdot\rangle\right)$ is a pairing over $\mathbb{K}$ and, as a consequence of the closed graph theorem, $B L(H)$ is the algebra of all linear operators on $H$ that have an adjoint with respect to $\langle\cdot, \cdot\rangle$, and so $F L(H)$ is the ideal of all linear operators on $H$ that have an adjoint with respect to $\langle\cdot, \cdot\rangle$ and are of finite rank. Since $A$ is a norm ideal on $H$, it follows that $A$ is a subalgebra of $B L(H)$ containing $F L(H)$ and, by [2; Theorem 4.3.7], $Q^{r}(A)=L(H)$.

For $T$ in $B L(H)$ and $F$ in $F L(H)$ we have

$$
\left\|L_{T}^{F L(H)}(F)\right\|=\|T F\| \leq\|T\|_{\infty}\|F\|,
$$

therefore $L_{T}^{F L(H)}:(F L(H),\|\cdot\|) \rightarrow(A,\|\cdot\|)$ is bounded and $\left\|L_{T}^{F L(H)}\right\| \leq$ $\|T\|_{\infty}$. Thus $B L(H) \subseteq Q_{b}^{r}(A)$ and $\|T\|_{r} \leq\|T\|_{\infty}$ for all $T$ in $B L(H)$. To prove the reverse inclusion, note that $F L(H)$ is the minimal ideal of $A$ and so $Q_{b}^{r}(A)$ is the subalgebra of $L(H)$ consisting of all $T$ in $L(H)$ such that

$$
L_{T}^{F L(H)}:(F L(H),\|\cdot\|) \rightarrow(A,\|\cdot\|)
$$

is bounded and $\|T\|_{r}=\left\|L_{T}^{F L(H)}\right\|$ for all $T$ in $Q_{b}^{r}(A)$. If $T$ is in $Q_{b}^{r}(A)$, then

$$
\begin{aligned}
\|T(x)\|\|x\| & =\|T(x) \otimes x\|=\|T(x \otimes x)\|=\left\|L_{T}^{F L(H)}(x \otimes x)\right\| \\
& \leq\left\|L_{T}^{F L(H)}\right\|\|x \otimes x\|=\left\|L_{T}^{F L(H)}\right\|\|x\|^{2}
\end{aligned}
$$

for all $x$ in $H$, hence $T$ lies in $B L(H)$ and $\|T\|_{\infty} \leq\|T\|_{r}$. Therefore, $\left(Q_{b}^{r}(A),\|\cdot\|_{r}\right)=\left(B L(H),\|\cdot\|_{\infty}\right)$.

Now, we shall show that $\left(Q_{b}^{l}(A),\|\cdot\|_{l}\right)=\left(B L(H),\|\cdot\|_{\infty}\right)$. Using the same reasoning as above, it is clear that for $T$ in $B L(H)$ the operator $R_{T}^{F L(H)}$ : $(F L(H),\|\cdot\|) \rightarrow(A,\|\cdot\|)$ is bounded and $\left\|R_{T}^{F L(H)}\right\| \leq\|T\|_{\infty}$. Therefore $B L(H) \subseteq Q_{b}^{l}(A)$ and $\|T\|_{l} \leq\|T\|_{\infty}$ for all $T$ in $B L(H)$. To prove the converse inclusion, fix $q$ in $Q_{b}^{l}(A)$. Then $\varrho:(F L(H),\|\cdot\|) \rightarrow(A,\|\cdot\|)$ defined by $\varrho(F)=F q$ is a bounded linear operator satisfying $\varrho(T F)=T \varrho(F)$ for all $F$ in $F L(H)$ and $T$ in $A$. Thus, the mapping $\lambda:\left(F L(H),\|\cdot\|^{*}\right) \rightarrow\left(A^{*},\|\cdot\|^{*}\right)$ defined by $\lambda(F):=\varrho\left(F^{*}\right)^{*}$ for all $F$ in $F L(H)$ is also a bounded linear operator satisfying $\lambda(F T)=\lambda(F) T$ for all $F$ in $F L(H)$ and $T$ in $A^{*}$. By $[2$; 
Proposition 2.2.1(iv)] and the definition of $Q_{b}^{r}\left(A^{*}\right)$ there exists $p$ in $Q_{b}^{r}\left(A^{*}\right)$ such that $\lambda(F)=p F$ for all $F$ in $F L(H)$. Now, applying the first part of the proof to the norm ideal $A^{*}$, we get the existence of $S$ in $B L(H)$ such that $\lambda(F)=L_{S}(F)$ for all $F$ in $F L(H)$. Consequently, $\varrho(F)=\lambda\left(F^{*}\right)^{*}=$ $\left(S F^{*}\right)^{*}=F S^{*}=R_{S^{*}}(F)$ for all $F$ in $F L(H)$, hence $\varrho=R_{S^{*}}^{F L(H)}$. Thus $Q_{b}^{l}(A)=B L(H)$. Now, using the same reasoning as before we see that $\|T\|_{\infty} \leq\left\|R_{T}^{F L(H)}\right\|$ for all $T$ in $B L(H)$. Therefore $\|\cdot\|_{l}=\|\cdot\|_{\infty}$.

Finally, from the definition of a symmetric bounded algebra of quotients it is clear that $\left(Q_{b}^{s}(A),\|\cdot\|_{s}\right)=\left(B L(H),\|\cdot\|_{\infty}\right)$.

As a consequence of Theorems 1 and 2, algebras of quotients with bounded evaluation of norm ideals on $H$ are subalgebras of $B L(H)$. Next, we will show that there is a useful relationship between the left or right algebras of quotients of bounded evaluation of a norm ideal and their adjoints.

Proposition 2. Let $(A,\|\cdot\|)$ be a norm ideal on $H$. Then

$$
\left(Q_{b e}^{r}(A), \mathbf{|} \cdot \mathbf{I}_{r}\right)^{*}=\left(Q_{b e}^{l}\left(A^{*}\right), \mathbf{|} \cdot \mathbf{I}_{l}\right) .
$$

Proof. Note that the algebra anti-isomorphism $*: A \rightarrow A^{*}$ leads to an isometric isomorphism

$$
*:(M(A),\|\cdot\|) \rightarrow\left(M\left(A^{*}\right),\|\cdot\|^{*}\right)
$$

defined by $\mathcal{T}^{*}(S)=\mathcal{T}\left(S^{*}\right)^{*}$ for all $\mathcal{T}$ in $M(A)$ and $S$ in $A^{*}$. For a nonzero ideal $I$ of $A$ it is clear that the equality $\left(I^{r}\right)^{*}=\left(I^{*}\right)^{l}$ holds. Furthermore, for $F$ in $B L(H)$ we have: $F I \subseteq A$ if, and only if, $I^{*} F^{*} \subseteq A^{*}$. In that case $E_{F}^{I^{r}}(\mathcal{T})=E_{F^{*}}^{\left(I^{*}\right)^{l}}\left(\mathcal{T}^{*}\right)^{*}$ for all $\mathcal{T}$ in $I^{r}$. Therefore, $\left\|E_{F}^{I^{r}}(\mathcal{T})\right\|=\left\|E_{F^{*}}^{\left(I^{*}\right)^{l}}\left(\mathcal{T}^{*}\right)\right\|^{*}$ for all $\mathcal{T}$ in $I^{r}$, and so $E_{F}^{I^{r}}$ is bounded if, and only if, $E_{F^{*}}^{\left(I^{*}\right)^{l}}$ is bounded. Moreover, in this case $\left\|E_{F}^{I^{r}}\right\|=\left\|E_{F^{*}}^{\left(I^{*}\right)^{l}}\right\|^{*}$. Consequently, $F$ lies in $Q_{b e}^{r}(A)$ if, and only if, $F^{*}$ lies in $Q_{b e}^{l}\left(A^{*}\right)$, and in that case $\left|F \mathbf{|}_{r}=\right| F^{*} \mathbf{I}_{l}$.

The above proposition makes it clear that the study of algebras of quotients with bounded evaluation of self-adjoint norm ideals focuses on right algebras.

Proposition 3. Let $(A,\|\cdot\|)$ be a norm ideal on $H$. Then $Q_{b e}^{r}(A)$ is a right ideal of $B L(H)$ and $\mathbf{|} \cdot \mathbf{I}_{r}$ is an algebra norm satisfying the following assertions:

(i) $\mid T \mathbf{|}_{r} \leq\|T\|$ for all $T$ in $A$,

(ii) $\|T\|_{\infty} \leq \mathbf{|} T \mathbf{|}_{r}$ for all $T$ in $Q_{b e}^{r}(A)$,

(iii) $\mathbf{|} x \otimes y \mathbf{|}_{r}=\|x\|\|y\|$ for all $x, y$ in $H$,

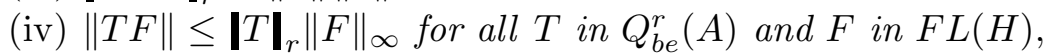

(v) $\mid T F \mathbf{|}_{r} \leq \mathbf{|} T \mathbf{|}_{r}\|F\|_{\infty}$ for all $T$ in $Q_{b e}^{r}(A)$ and $F$ in $B L(H)$. 
Proof. By Theorems 1 and 2, $Q_{b e}^{r}(A)$ is a subalgebra of $B L(H)$ containing $A$ and $|\cdot|_{r}$ is an algebra seminorm on $Q_{b e}^{r}(A)$ satisfying (i) and (ii). As a consequence of (ii), $|\cdot| \mathbf{I}_{r}$ is in fact a norm on $Q_{b e}^{r}(A)$.

(iii) Using (ii) and (i) we see that for $x, y$ in $H$,

$$
\|x\|\|y\|=\|x \otimes y\|_{\infty} \leq \mathbf{|} x \otimes y \mathbf{I}_{r} \leq\|x \otimes y\|=\|x\|\|y\|,
$$

hence $\mathbf{|} x \otimes y \mathbf{|}_{r}=\|x\|\|y\|$.

(iv) For $T$ in $Q_{b e}^{r}(A)$ and $F$ in $F L(H)$ we have

$$
\begin{aligned}
\|T F\| & =\left\|E_{T}^{F L(H)^{r}}\left(R_{F}\right)\right\| \leq\left\|E_{T}^{F L(H)^{r}}\right\|\left\|R_{F}\right\| \\
& \leq\left\|E_{T}^{F L(H)^{r}}\right\|\|F\|_{\infty}=\mid \mathbf{|} T \mathbf{I}_{r}\|F\|_{\infty} .
\end{aligned}
$$

Finally, we shall prove that $Q_{b e}^{r}(A)$ is a right ideal of $B L(H)$ and that (v) is satisfied. Since $F L(H)$ is a nonzero ideal of $B L(H)$ it follows that the inclusion $F L(H)^{r} R_{F} \subseteq F L(H)^{r}$ holds for every $F$ in $B L(H)$. Furthermore, for $\mathcal{T}$ in $F L(H)^{r}$ and $F$ in $B L(H)$ we see that

$$
\left\|\left(\mathcal{T} R_{F}\right)(T)\right\|=\|\mathcal{T}(T F)\| \leq\|\mathcal{T}\|\|T F\| \leq\|\mathcal{T}\|\|T\|\|F\|_{\infty}
$$

for all $T$ in $A$, hence $\left\|\mathcal{T} R_{F}\right\| \leq\|\mathcal{T}\|\|F\|_{\infty}$.

Now, for $T$ in $Q_{b e}^{r}(A), F$ in $B L(H)$, and $\mathcal{T}$ in $F L(H)^{r}$ we have

$$
\begin{aligned}
\left\|E_{T F}^{F L(H)^{r}}(\mathcal{T})\right\| & =\|\mathcal{T}(T F)\|=\left\|\mathcal{T} R_{F}(T)\right\|=\left\|E_{T}^{F L(H)^{r}}\left(\mathcal{T} R_{F}\right)\right\| \\
& \leq\left\|E_{T}^{F L(H)^{r}}\right\|\left\|\mathcal{T} R_{F}\right\| \leq\left\|E_{T}^{F L(H)^{r}}\right\|\|\mathcal{T}\|\|F\|_{\infty},
\end{aligned}
$$

hence $E_{T F}^{F L(H)^{r}}$ is bounded and $\left\|E_{T F}^{F L(H)^{r}}\right\| \leq\left\|E_{T}^{F L(H)^{r}}\right\|\|F\|_{\infty}$, and so $T F$ lies in $Q_{b e}^{r}(A)$ and $\mid T F \mathbf{|}_{r} \leq \mathbf{|} T \mathbf{|}_{r}\|F\|_{\infty}$.

We now study the algebras of quotients with bounded evaluation of the ideal of all compact operators on $H$. Recall that an operator $F$ on $H$ is called compact if $F\left(B_{H}\right)$ is relatively compact in the norm topology, where $B_{H}$ is the closed unit ball of $H$. The set $K L(H)$ of all compact operators on $H$ is a self-adjoint closed ideal of $B L(H)$ and $F L(H)$ is dense in $\left(K L(H),\|\cdot\|_{\infty}\right)$. Also we recall that the strong operator topology on $B L(H)$ is the topology of pointwise convergence, and that for this topology the left and right multiplications by a fixed operator are bounded.

TheOREm 3. Let $K L(H)$ be the ideal of all compact operators on $H$. Then

$$
\begin{aligned}
\left(Q_{b e}^{r}(K L(H)), \mathbf{|} \cdot \mathbf{I}_{r}\right) & =\left(Q_{b e}^{l}(K L(H)), \mathbf{|} \cdot \mathbf{l}_{l}\right)=\left(Q_{b e}^{s}(K L(H)), \mathbf{|} \cdot \mathbf{I}_{s}\right) \\
& =\left(B L(H),\|\cdot\|_{\infty}\right) .
\end{aligned}
$$

Proof. We begin by proving that $\left(Q_{b e}^{r}(K L(H)), \mathbf{|} \cdot \mathbf{I}_{r}\right)=\left(B L(H),\|\cdot\|_{\infty}\right)$. By Proposition 3(ii), $Q_{b e}^{r}(K L(H))$ is a right ideal of $B L(H)$ and $\|T\|_{\infty} \leq$ $\mathbf{|} T \mathbf{|}_{r}$ for all $T$ in $Q_{b e}^{r}(K L(H))$. We claim that if $\mathcal{T}$ is in $M(B L(H))$ and $\mathcal{T}^{K L(H)}$ denotes the restriction of $\mathcal{T}$ to $K L(H)$, then $\|\mathcal{T}\|_{\infty}=\left\|\mathcal{T}^{K L(H)}\right\|_{\infty}$. 
Let $\mathcal{T}$ be in $M(B L(H))$. For each positive number $\varepsilon$ we can take $F$ in the closed unit ball $B_{B L(H)}$ of $B L(H)$ and $x$ in $B_{H}$ such that $\|\mathcal{T}\|_{\infty}-\varepsilon<$ $\|\mathcal{T}(F)(x)\|$. It is well known that the closed unit ball $B_{K L(H)}$ of $K L(H)$ is strongly dense in $B_{B L(H)}$ (see [10; Lemma 3.3.2]). Therefore we can assume the existence of a net $\left\{F_{\lambda}\right\}_{\lambda \in \Lambda}$ in $B_{K L(H)}$ which is strongly convergent to $F$. By the separate strong continuity of the product of $B L(H)$, it follows that $\left\{\mathcal{T}\left(F_{\lambda}\right)\right\}_{\lambda \in \Lambda}$ is strongly convergent to $\mathcal{T}(F)$. Consequently, $\left\{\left\|\mathcal{T}\left(F_{\lambda}\right)(x)\right\|\right\}_{\lambda \in \Lambda}$ converges to $\|\mathcal{T}(F)(x)\|$. Therefore, there is $\lambda_{0}$ in $\Lambda$ such that $\|\mathcal{T}\|_{\infty}-\varepsilon<\left\|\mathcal{T}\left(F_{\lambda}\right)(x)\right\|$ for all $\lambda \geq \lambda_{0}$. Thus,

$$
\|\mathcal{T}\|_{\infty}-\varepsilon<\left\|\mathcal{T}\left(F_{\lambda_{0}}\right)(x)\right\| \leq\left\|\mathcal{T}\left(F_{\lambda_{0}}\right)\right\|_{\infty} \leq\left\|\mathcal{T}^{K L(H)}\right\|_{\infty} .
$$

Since $\varepsilon$ is an arbitrary positive number, we obtain $\|\mathcal{T}\|_{\infty}=\left\|\mathcal{T}^{K L(H)}\right\|_{\infty}$.

Now, taking into account the inclusions

$$
F L(H)^{r} \subseteq M(K L(H)) \subseteq M(B L(H)) \subseteq B L(B L(H))
$$

(where $F L(H)^{r}$ is the ideal of $M(K L(H))$ generated by the set $\left\{R_{F}: F \in\right.$ $F L(H)\})$ and the claim which was proven above, it follows that for $T$ in $B L(H)$ and $\mathcal{T}$ in $F L(H)^{r}$,

$$
\left\|E_{T}^{F L(H)^{r}}(\mathcal{T})\right\|_{\infty}=\|\mathcal{T}(T)\|_{\infty} \leq\|\mathcal{T}\|_{\infty}\|T\|_{\infty}=\left\|\mathcal{T}^{K L(H)}\right\|_{\infty}\|T\|_{\infty},
$$

hence $E_{T}^{F L(H)^{r}}$ is bounded and $\left\|E_{T}^{F L(H)^{r}}\right\|_{\infty} \leq\|T\|_{\infty}$, and so $T$ lies in $Q_{b e}^{r}(K L(H))$ and $\mid T \mathbf{|}_{r} \leq\|T\|_{\infty}$.

Finally, by Proposition $2,\left(Q_{b e}^{l}(K L(H)), \mathbf{|} \cdot \mathbf{I}_{l}\right)=\left(B L(H),\|\cdot\|_{\infty}\right)$ because $\left(K L(H),\|\cdot\|_{\infty}\right)^{*}=\left(K L(H),\|\cdot\|_{\infty}\right)$. Thus also $\left(Q_{b e}^{s}(K L(H)),|\cdot| \mathbf{I}_{s}\right)=$ $\left(B L(H),\|\cdot\|_{\infty}\right)$.

For the remainder of this section, we will turn our attention to the study of the algebras of quotients with bounded evaluation of the Schatten $p$ ideals. We will include some aspects of these ideals that are pertinent to the development of this paper and that can be found for example in [10], [11], [13], and [14]. Recall that an operator $T$ in $B L(H)$ has a Schmidt representation if there are orthonormal families $\left\{x_{i}\right\}_{i \in I},\left\{y_{i}\right\}_{i \in I}$ of vectors of $H$ and a scalar family $\left\{\sigma_{i}\right\}_{i \in I}$ such that $T=\sum_{i \in I} \sigma_{i} x_{i} \otimes y_{i}$. The Schatten ideal $\mathcal{C}_{p}(H)(1 \leq p<\infty)$ is defined as the set of all operators $T$ in $B L(H)$ which have a Schmidt representation $T=\sum_{i \in I} \sigma_{i} x_{i} \otimes y_{i}$ such that

$$
\sum_{i \in I}\left|\sigma_{i}\right|^{p}<\infty
$$

and the Schatten norm $\|\cdot\|_{p}$ of $T$ is defined by

$$
\|T\|_{p}=\left(\sum_{i \in I}\left|\sigma_{i}\right|^{p}\right)^{1 / p} .
$$


This definition does not depend on the Schmidt representation chosen. $\left(\mathcal{C}_{p}(H),\|\cdot\|_{p}\right)$ is a Banach algebra which is a self-adjoint norm ideal on $H$ and the involution $*$ is $\|\cdot\|_{p}$-isometric. Moreover, $F L(H) \subseteq \mathcal{C}_{p}(H) \subseteq K L(H)$ and $F L(H)$ is dense in $\left(\mathcal{C}_{p}(H),\|\cdot\|_{p}\right)$. Also, $\mathcal{C}_{p}(H) \subseteq \mathcal{C}_{q}(H)$ for $1 \leq p \leq q<\infty$ and $\|T\|_{q} \leq\|T\|_{p}$ for all $T$ in $\mathcal{C}_{p}(H)$. The Schatten ideal $\mathcal{C}_{1}(H)$ is called the ideal of trace class operators, which is justified by the fact that $\mathcal{C}_{1}(H)$ is the domain of the trace function tr, defined by

$$
\operatorname{tr}(T)=\sum_{\lambda \in \Lambda}\left\langle T\left(e_{\lambda}\right), e_{\lambda}\right\rangle
$$

for all $T$ in $\mathcal{C}_{1}(H)$, where $\left\{e_{\lambda}\right\}_{\lambda \in \Lambda}$ is an arbitrary orthonormal basis in $H$ (again, this definition does not depend on the basis selected). Furthermore, the mapping $\operatorname{tr}: \mathcal{C}_{1}(H) \rightarrow \mathbb{K}$ is a continuous commutative linear form satisfying $|\operatorname{tr}(T)| \leq\|T\|_{1}$ for all $T$ in $\mathcal{C}_{1}(H)$.

Recall that a bounded linear operator $T$ on $H$ is called positive if $T^{*}=T$ and $\langle T(x), x\rangle \geq 0$ for all $x$ in $H$. If $T$ is a positive operator, then $T$ has a unique positive square root, which will be denoted by $T^{1 / 2}$. The absolute value of a bounded linear operator $F$ is defined as the positive square root of $F^{*} F$ and will be denoted by $[F]$, that is, $[F]=\left(F^{*} F\right)^{1 / 2}$. Finally, recall that an operator $W$ on $H$ is said to be partially isometric if $W W^{*} W=W$.

Theorem 4. Let $\mathcal{C}_{p}(H)(1 \leq p<\infty)$ be the Schatten $p$-ideal. Then

$$
\begin{aligned}
\left(Q_{b e}^{r}\left(\mathcal{C}_{p}(H)\right), \mathbf{|} \cdot \mathbf{I}_{r}\right) & =\left(Q_{b e}^{l}\left(\mathcal{C}_{p}(H)\right), \mathbf{|} \cdot \mathbf{I}_{l}\right)=\left(Q_{b e}^{s}\left(\mathcal{C}_{p}(H)\right), \mathbf{|} \cdot \mathbf{I}_{s}\right) \\
& =\left(\mathcal{C}_{p}(H),\|\cdot\|_{p}\right) .
\end{aligned}
$$

Proof. We first prove that

$$
\left(Q_{b e}^{r}\left(\mathcal{C}_{p}(H)\right), \mathbf{I} \cdot \mathbf{I}_{r}\right)=\left(\mathcal{C}_{p}(H),\|\cdot\|_{p}\right) .
$$

By Proposition 3, $Q_{b e}^{r}\left(\mathcal{C}_{p}(H)\right)$ is a right ideal of $B L(H)$. Let $T$ be a positive operator in $Q_{b e}^{r}\left(\mathcal{C}_{p}(H)\right)$. We claim that $T$ belongs to $\mathcal{C}_{p}(H)$ and $\|T\|_{p} \leq \mathbf{|} T \mathbf{|}_{r}$. Again by Proposition 3(iv) we have

$$
\|F T\|_{p}=\left\|T F^{*}\right\|_{p} \leq \mathbf{|} T \mathbf{|}_{r}\|F\|_{\infty}
$$

for all $F$ in $F L(H)$. Choose a natural number $n$ such that $p \leq 2^{n}$. We shall prove that

$$
\left\|F T^{2^{k}}\right\|_{2^{n-k}} \leq \mid \mathbf{|} \mathbf{|}_{r}^{2^{k}}\|F\|_{\infty}
$$

for all $F$ in $F L(H)$ and $k \in\{0,1, \ldots, n\}$. The proof is by induction on $k$. For $k=0$ the result follows directly from (5) and from the fact that $\|\cdot\|_{2^{n}} \leq\|\cdot\|_{p}$. Now suppose that (6) holds for some $k \in\{0,1, \ldots, n-1\}$. If for a fixed $F$ in $F L(H)$ we denote by $P$ the orthogonal projection from $H$ onto the finite-dimensional subspace $T^{2^{k+1}} F^{*}(H)$, then

$$
F T^{2^{k+1}}=\left(T^{2^{k+1}} F^{*}\right)^{*}=\left(P T^{2^{k+1}} F^{*}\right)^{*}=F T^{2^{k+1}} P,
$$


and so

$$
\left\|F T^{2^{k+1}}\right\|_{2^{n-k-1}}=\left\|F T^{2^{k+1}} P\right\|_{2^{n-k-1}} .
$$

By [11; Theorem 15.5.9], $R S$ belongs to $\mathcal{C}_{2^{n-k-1}}(H)$ whenever $R$ and $S$ are in $\mathcal{C}_{2^{n-k}}(H)$, and in that case $\|R S\|_{2^{n-k-1}} \leq\|R\|_{2^{n-k}}\|S\|_{2^{n-k}}$. Thus,

$$
\begin{aligned}
\left\|F T^{2^{k+1}}\right\|_{2^{n-k-1}} & \leq\left\|F T^{2^{k}}\right\|_{2^{n-k}}\left\|T^{2^{k}} P\right\|_{2^{n-k}} \leq\left|T \mathbf{|}_{r}^{2^{k}}\|F\|_{\infty}\right| T \mathbf{|}_{r}^{2^{k}}\|P\|_{\infty} \\
& =\mid T \mathbf{|}_{r}^{2^{k+1}}\|F\|_{\infty},
\end{aligned}
$$

proving (6) for $k+1$, and concluding the induction. Now, taking $k=n$ in (6), we have $\left\|F T^{2^{n}}\right\|_{1} \leq \mid T \mathbf{|}_{r}^{2^{n}}\|F\|_{\infty}$ for all $F$ in $F L(H)$, and consequently $\left|\operatorname{tr}\left(F T^{2^{n}}\right)\right| \leq \mid T \mathbf{|}_{r}^{2^{n}}\|F\|_{\infty}$ for all $F$ in $F L(H)$. By $[11 ; 6.4 .1$ and Theorem 22.1.9], $T^{2^{n}}$ is a trace class operator and so $T^{2^{n}}$ is compact. Consequently, $T$ is also compact and so can be diagonalized. Thus, there is an orthonormal family $\left\{e_{i}\right\}_{i \in I}$ and a nonnegative family $\left\{\lambda_{i}\right\}_{i \in I}$ which vanishes at infinity such that $T=\sum_{i \in I} \lambda_{i} e_{i} \otimes e_{i}$. Now, consider the projection $P_{\Gamma}=\sum_{k=1}^{n} e_{i_{k}} \otimes e_{i_{k}}$ for every finite subset $\Gamma=\left\{i_{1}, \ldots, i_{n}\right\}$ of $I$. Then, from (5), we have $\sum_{k=1}^{n} \lambda_{i_{k}}^{p}=\left\|P_{\Gamma} T\right\|_{p}^{p} \leq|T|_{r}^{p}\left\|P_{\Gamma}\right\|_{\infty}^{p}=|T|_{r}^{p}$. Therefore, $T$ lies in $\mathcal{C}_{p}(H)$ and $\|T\|_{p} \leq \mid T \mathbf{|}_{r}$.

Now, let $T$ be an arbitrary operator in $Q_{b e}^{r}\left(\mathcal{C}_{p}(H)\right)$. Due to the polar decomposition of $T^{*}$, there exists a unique partial isometry $W$ such that $T^{*}=W\left[T^{*}\right]$; furthermore, $\left[T^{*}\right]=W^{*} T^{*}$. Since $Q_{b e}^{r}\left(\mathcal{C}_{p}(H)\right)$ is a right ideal of $B L(H)$, it follows that $\left[T^{*}\right]=T W$ belongs to $Q_{b e}^{r}\left(\mathcal{C}_{p}(H)\right)$. By Proposition $3(\mathrm{v})$ we also see that

$$
\mathbf{I}\left[T^{*}\right] \mathbf{I}_{r}=\mathbf{|} T W \mathbf{|}_{r} \leq \mathbf{I} T \mathbf{|}_{r}\|W\|_{\infty}=\mathbf{|} T \mathbf{|}_{r} .
$$

Since $\left[T^{*}\right]$ is positive, from the first part of the proof we conclude that $\left[T^{*}\right]$ belongs to $\mathcal{C}_{p}(H)$ and $\left\|\left[T^{*}\right]\right\|_{p} \leq\left.|| T\right|_{r}$. Now, taking into account the equality $T^{*}=W\left[T^{*}\right]$, we can conclude that $T^{*}$ (hence $T$ ) belongs to $\mathcal{C}_{p}(H)$. Furthermore, since

$$
\|T\|_{p}=\left\|T^{*}\right\|_{p}=\left\|W\left[T^{*}\right]\right\|_{p} \leq\left\|\left[T^{*}\right]\right\|_{p} \leq \mathbf{|} T \mathbf{|}_{r},
$$

it follows that $\|T\|_{p} \leq \mid T \mathbf{|}_{r}$. The converse inequality is valid, as stated in Proposition 3(i).

Finally, according to Proposition $2,\left(Q_{b e}^{l}\left(\mathcal{C}_{p}(H)\right), \mathbf{|} \cdot \mathbf{l}_{l}\right)=\left(\mathcal{C}_{p}(H),\|\cdot\|_{p}\right)$ since $\left(\mathcal{C}_{p}(H),\|\cdot\|_{p}\right)^{*}=\left(\mathcal{C}_{p}(H),\|\cdot\|_{p}\right)$. Thus, $\left(Q_{b e}^{s}\left(\mathcal{C}_{p}(H)\right), \mathbf{|} \cdot \mathbf{I}_{s}\right)=\left(\mathcal{C}_{p}(H)\right.$, $\left.\|\cdot\|_{p}\right)$ as well.

\section{Algebras of quotients with bounded evaluation for totally} prime algebras. Totally prime algebras were introduced in [4] to provide the nonassociative extension of the determination of the extended centroid for ultraprime algebras given in [7]. We recall that a normed algebra $A$ is totally prime if there exists a positive number $K$ such that $K\|a\|\|b\| \leq\left\|N_{a, b}\right\|$ 
for all $a, b$ in $A$, where $N_{a, b}$ denotes the bilinear mapping from $M(A) \times M(A)$ into $A$ defined by $N_{a, b}(F, G)=F(a) G(b)$ for all $F, G$ in $M(A)$.

Lemma 2. Let $A$ be a totally prime algebra, and assume that $K$ is a positive number such that

$$
K\|a\|\|b\| \leq\left\|N_{a, b}\right\|
$$

for all $a, b$ in $A$. Let $q$ be in $Q_{b e}^{r}(A)$ and let $I$ be a nonzero ideal of $A$ such that $q I \subseteq A$ and $E_{q}^{I^{r}}$ is bounded. If $J$ is a nonzero ideal of $A$ such that $q J \subseteq A$, then $E_{q}^{J^{r}}$ is bounded and $K\left\|E_{q}^{J^{r}}\right\| \leq\left\|E_{q}^{I^{r}}\right\|$.

Proof. Let $J$ be a nonzero ideal of $A$ such that $q J \subseteq A$. For $x$ in $I$ with $\|x\|=1, F$ in $J^{r}$, and $S, T$ in $M(A)$ we have

$$
N_{F(q), x}(S, T)=S F(q) T(x)=R_{T(x)} S F(q)=E_{q}^{I^{r}}\left(R_{T(x)} S F\right),
$$

hence

$$
\left\|N_{F(q), x}(S, T)\right\|=\left\|E_{q}^{I^{r}}\left(R_{T(x)} S F\right)\right\| \leq\left\|E_{q}^{I^{r}}\right\|\|T\|\|S\|\|F\|,
$$

and so

$$
\left\|N_{F(q), x}\right\| \leq\left\|E_{q}^{I^{r}}\right\|\|F\| .
$$

Since $A$ is a totally prime algebra, it follows that

$$
K\|F(q)\| \leq\left\|E_{q}^{I^{r}}\right\|\|F\|,
$$

and so $K\left\|E_{q}^{J^{r}}(F)\right\| \leq\left\|E_{q}^{I^{r}}\right\|\|F\|$. Therefore $E_{q}^{J^{r}}$ is bounded and $K\left\|E_{q}^{J^{r}}\right\| \leq$ $\left\|E_{q}^{I^{r}}\right\|$.

TheOREM 5. Let $A$ be a totally prime algebra, and assume that $K$ is a positive number such that

$$
K\|a\|\|b\| \leq\left\|N_{a, b}\right\|
$$

for all $a, b$ in $A$. Then

(i) $\left(Q_{b e}^{r}(A), \mathbf{|} \cdot \mathbf{I}_{r}\right)$ is a normed algebra, and the inclusions of $A$ into $Q_{b e}^{r}(A)$ and of $M(A)$ into $M\left(Q_{b e}^{r}(A)\right)$ are topological. Precisely, $K\|a\| \leq$ $|a|_{r} \leq\|a\|$ for all $a$ in $A$, and $K\|F\| \leq \mid F \mathbf{|}_{r} \leq\|F\|$ for all $F$ in $M(A)$.

(ii) $\left(Q_{b e}^{r}(A), \mathbf{|} \cdot \mathbf{I}_{r}\right)$ is a totally prime algebra. Precisely,

$$
K^{2}\left|p \mathbf{I}_{r}\right| q \mathbf{|}_{r} \leq \mathbf{|} N_{p, q}^{I^{r} \times J^{r}} \mathbf{I}_{r}
$$

for all $p, q$ in $Q_{b e}^{r}(A)$ and nonzero ideals $I, J$ of $A$ such that $p I \subseteq A$ and $q J \subseteq A$, where $N_{p, q}^{I^{r} \times J^{r}}$ denotes the restriction of $N_{p, q}$ to $I^{r} \times J^{r}$.

(iii) If $(Q, \rrbracket \cdot \rrbracket)$ is a normed algebra such that $Q$ is a subalgebra of $Q^{r}(A)$ containing $A$ and the inclusions of $A$ into $Q$ and of $M(A)$ into $M(Q)$ are topological, then $Q$ is contained in $Q_{b e}^{r}(A)$ and this inclusion is continuous.

Proof. (i) By Theorem 1, $Q_{b e}^{r}(A)$ is an algebra extension of $A$ and $\mathbf{I} \cdot \mathbf{I}_{r}$ is an algebra seminorm on $Q_{b e}^{r}(A)$ such that $|a|_{r} \leq\|a\|$ for all $a$ in $A$. Let 
$q$ in $Q_{b e}^{r}(A)$ satisfy $\mid q \mathbf{|}_{r}=0$. By Lemma $2, E_{q}^{I^{r}}=0$ (hence $q I=0$ ) for all nonzero ideals $I$ of $A$ such that $q I \subseteq A$, and so $q=0$. Therefore, $\mathbf{I} \cdot \mathbf{I}_{r}$ is a norm on $Q_{b e}^{r}(A)$. Let $a$ be in $A$ and let $I$ be a nonzero ideal of $A$. For $x$ in $I$ with $\|x\|=1$ and $F, G$ in $M(A)$ we have

$$
N_{a, x}(F, G)=F(a) G(x)=R_{G(x)} F(a)=E_{a}^{I^{r}}\left(R_{G(x)} F\right),
$$

hence

$$
\left\|N_{a, x}(F, G)\right\|=\left\|E_{a}^{I^{r}}\left(R_{G(x)} F\right)\right\| \leq\left\|E_{a}^{I^{r}}\right\|\|G\|\|F\|,
$$

and so

$$
\left\|N_{a, x}\right\| \leq\left\|E_{a}^{I^{r}}\right\|
$$

Since $A$ is a totally prime algebra it follows that $K\|a\| \leq\left\|E_{a}^{I^{r}}\right\|$. Now, taking the infimum over $I$ we obtain $K\|a\| \leq|a|_{r}$.

By Theorem 1, $M(A)$ is continuously embedded in $M\left(Q_{b e}^{r}(A)\right)$ and $|F|_{r} \leq\|F\|$ for all $F$ in $M(A)$. Our objective now is to show that this inclusion is topological. Let $F$ be in $M(A)$. Then

$$
K\|F(a)\| \leq \mathbf{|} F(a) \mathbf{I}_{r} \leq\left|F \mathbf{|}_{r}\right| a \mathbf{|}_{r} \leq \mathbf{|} F \mathbf{I}_{r}\|a\|
$$

for all $a$ in $A$. Therefore $K\|F\| \leq \mid F \mathbf{|}_{r}$.

(ii) We fix $p, q$ in $Q_{b e}^{r}(A)$ and we consider nonzero ideals $I, J$ of $A$ such that $p I \subseteq A$ and $q J \subseteq A$. For $F$ in $I^{r}, G$ in $J^{r}$, and $S, T$ in $M(A)$ we have

$$
N_{F(p), G(q)}(S, T)=S F(p) T G(q)=N_{p, q}^{I^{r} \times J^{r}}(S F, T G) .
$$

Hence

$$
\begin{array}{r}
K\left\|N_{F(p), G(q)}(S, T)\right\|=K\left\|N_{p, q}^{I^{r} \times J^{r}}(S F, T G)\right\| \leq \mathbf{|} N_{p, q}^{I^{r} \times J^{r}}(S F, T G) \mathbf{I}_{r} \\
\leq \mathbf{|} N_{p, q}^{I^{r} \times J^{r}} \mathbf{I}_{r}\left|S \mathbf{|}_{r}\right| F \mathbf{|}_{r}\left|T \mathbf{I}_{r}\right| G \mathbf{|}_{r} \leq \mathbf{|} N_{p, q}^{I^{r} \times J^{r}} \mathbf{I}_{r}\|S\|\|F\|\|T\|\|G\|,
\end{array}
$$

and so

$$
K\left\|N_{F(p), G(q)}\right\| \leq \mathbf{I} N_{p, q}^{I^{r} \times J^{r}} \mathbf{I}_{r}\|F\|\|G\|
$$

Therefore,

$$
K^{2}\|F(p)\|\|G(q)\| \leq \mathbf{I} N_{p, q}^{I^{r} \times J^{r}} \mathbf{I}_{r}\|F\|\|G\|,
$$

since $A$ is a totally prime algebra. From the last inequality it follows that

$$
K^{2}\left\|E_{p}^{I^{r}}\right\|\left\|E_{q}^{J^{r}}\right\| \leq \mid N_{p, q}^{I^{r} \times J^{r}} \mathbf{I}_{r}
$$

and so

$$
K^{2}\left|p \mathbf{I}_{r}\right| q \mathbf{|}_{r} \leq \mathbf{|} N_{p, q}^{I^{r} \times J^{r}} \mathbf{I}_{r}
$$

(iii) Let $(Q, \rrbracket \cdot \rrbracket)$ be a normed algebra such that $Q$ is a subalgebra of $Q^{r}(A)$ containing $A$ and assume the existence of positive numbers $\alpha, \beta, \gamma, \delta$ such that $\alpha\|a\| \leq \llbracket a \rrbracket \leq \beta\|a\|$ for all $a$ in $A$, and $\gamma\|F\| \leq \rrbracket F \rrbracket \leq \delta\|F\|$ for all $F$ in $M(A)$. Let $q$ be in $Q$, and assume that $I$ is a nonzero ideal of $A$ such that $q I \subseteq A$. Then for all $F$ in $I^{r}$ we have

$$
\left\|E_{q}^{I^{r}}(F)\right\|=\|F(q)\| \leq \alpha^{-1} \llbracket F(q) \rrbracket \leq \alpha^{-1} \rrbracket F \rrbracket \rrbracket q \rrbracket \leq \alpha^{-1} \delta\|F\| \rrbracket q \rrbracket,
$$


hence $E_{q}^{I^{r}}$ is bounded and $\left\|E_{q}^{I^{r}}\right\| \leq \alpha^{-1} \delta \rrbracket q \rrbracket$, and so $q$ lies in $Q_{b e}^{r}(A)$ and $\mid \mathbf{|} q \mathbf{|}_{r} \leq \alpha^{-1} \delta \rrbracket q \rrbracket$. Therefore $Q$ is continuously embedded in $Q_{b e}^{r}(A)$.

As a consequence of the above theorem, we can obtain a topological characterization of the subalgebras of $\left(Q_{b e}^{r}(A), \mathbf{|} \cdot \mathbf{I}_{r}\right)$ whenever $A$ is a totally prime algebra.

Corollary 1. Let $A$ be a totally prime algebra.

(i) If $Q$ is a normed subalgebra of $Q_{b e}^{r}(A)$ containing $A$, then the inclusions of $A$ into $Q$ and of $M(A)$ into $M(Q)$ are topological, and there exists a positive number $K^{\prime}$ such that

$$
K^{\prime}\left|p \mathbf{I}_{r}\right| q \mathbf{|}_{r} \leq \mathbf{|} N_{p, q}^{I^{r} \times J^{r}} \mathbf{I}_{r}
$$

for all $p, q$ in $Q$ and nonzero ideals $I, J$ of $A$ such that $p I \subseteq A$ and $q J \subseteq A$.

(ii) If $(Q, \rrbracket \cdot \rrbracket)$ is a normed algebra such that $Q$ is a subalgebra of $Q^{r}(A)$ containing $A$ and the inclusions of $A$ into $Q$ and of $M(A)$ into $M(Q)$ are topological, and there exists a positive number $K^{\prime}$ such that

$$
K^{\prime} \rrbracket p \rrbracket \rrbracket q \rrbracket \leq \llbracket N_{p, q}^{I^{r} \times J^{r}} \rrbracket
$$

for all $p, q$ in $Q$ and nonzero ideals $I, J$ of $A$ such that $p I \subseteq A$ and $q J \subseteq A$, then $Q$ is contained in $Q_{b e}^{r}(A)$ and this inclusion is topological.

Proof. (i) Assume that $K$ is a positive number such that $K\|a\|\|b\| \leq$ $\left\|N_{a, b}\right\|$ for all $a, b$ in $A$. According to Theorem $5(\mathrm{i}), K\|a\| \leq \mid a \mathbf{|}_{r} \leq\|a\|$ for all $a$ in $A$ and $K\|F\| \leq|F|_{r} \leq\|F\|$ for all $F$ in $M(A)$. Consequently, the inclusion of $A$ into $Q$ is topological. Next, we show that the inclusion of $M(A)$ into $M(Q)$ is also topological. For $F$ in $M(A)$, if $F^{Q}$ denotes the operator $F$ considered in $M(Q)$, then $\left.\left|F^{Q} \mathbf{I}_{r} \leq\right| F\right|_{r} \leq\|F\|$. On the other hand,

$$
K\|F(a)\| \leq \mathbf{|} F(a) \mathbf{I}_{r}=\mathbf{|} F^{Q}(a) \mathbf{I}_{r} \leq \mathbf{|} F^{Q} \mathbf{I}_{r} \mid a \mathbf{I}_{r} \leq \mathbf{|} F^{Q} \mathbf{I}_{r}\|a\|
$$

for all $a$ in $A$ and so $K\|F\| \leq \mid F^{Q} \mathbf{I}_{r}$. Finally, Theorem 5(ii) concludes the proof of (i).

(ii) Let $(Q, \rrbracket \cdot \rrbracket)$ be a normed algebra as in part (ii) of the statement. By Theorem $5, Q$ is continuously embedded in $Q_{b e}^{r}(A)$. Consider $\alpha, \beta, \gamma, \delta$ as at the end of the proof of Theorem 5. Fix $q$ in $Q$, and assume that $I$ is a nonzero ideal of $A$ such that $q I \subseteq A$. For each $a$ in $A$ with $\llbracket a \rrbracket=1$ and $0<\varepsilon<1$ we can choose $F$ in $I^{r}$ with $\llbracket F \rrbracket=1$ and $G$ in $M(A)$ with $\llbracket G \rrbracket=1$ such that $\varepsilon K^{\prime} \rrbracket q \rrbracket \leq \llbracket N_{q, a}(F, G) \rrbracket$. Therefore,

$$
\begin{aligned}
\varepsilon K^{\prime} \rrbracket q \rrbracket & \leq \rrbracket F(q) G(a) \rrbracket \leq \rrbracket F(q) \rrbracket \rrbracket G(a) \rrbracket \leq \beta\|F(q)\| \\
& \leq \beta\left\|E_{q}^{I^{r}}\right\|\|F\| \leq \beta \gamma^{-1}\left\|E_{q}^{I^{r}}\right\| .
\end{aligned}
$$

This shows that $K^{\prime} \rrbracket q \rrbracket \leq \beta \gamma^{-1}\left\|E_{q}^{I^{r}}\right\|$. Consequently, $K^{\prime} \rrbracket q \rrbracket \leq \beta \gamma^{-1} \mid q \mathbf{I}_{r}$, as required. 
Recall that the extended centroid $C(A)$ of a prime algebra $A$ is a field extension of $\mathbb{K}$ via the canonical homomorphism $\lambda \mapsto \lambda \mathbb{1}$ of $\mathbb{K}$ into $C(A)$. If $C(A)$ is equal to $\mathbb{K}$, then $A$ is said to be centrally closed. Totally prime real algebras have extended centroid equal to $\mathbb{R}$ or $\mathbb{C}$, and therefore totally prime complex algebras are centrally closed [4; Theorem 2 and Remark 2]. For non-centrally closed real algebras, the above theorem may be completed with the following result:

Proposition 2. If $A$ is a totally prime real algebra with extended centroid $\mathbb{C}$, then $Q_{b e}^{r}(A)$ is a complex subalgebra of $Q^{r}(A)$ and there exists a complex algebra norm on $Q_{b e}^{r}(A)$ equivalent to $\mid \cdot \mathbf{I}_{r}$.

Proof. Let $A$ be a totally prime real algebra with extended centroid $\mathbb{C}$. Suppose that $K$ is a positive number such that $K\|a\|\|b\| \leq\left\|N_{a, b}\right\|$ for all $a, b$ in $A$, and denote by $i$ the imaginary unit of $C(A)$. Fix a nonzero ideal $I$ of $A$ such that $i I \subseteq A$, and note that $F(i x)=i F(x)$ for all $F$ in $M(A)$ and $x$ in $I$. As a consequence, $N_{i x, y}=N_{x, i y}$ for all $x, y$ in $I$, and therefore

$$
K\|i x\|\|y\| \leq\left\|N_{i x, y}\right\|=\left\|N_{x, i y}\right\| \leq\|x\|\|i y\|
$$

for all $x, y$ in $I$. It follows that the mapping $\iota: I \rightarrow A$ defined by $\iota(x):=i x$ is continuous. Now, given $q$ in $Q_{b e}^{r}(A)$, consider a nonzero ideal $J$ of $A$ such that $q J \subseteq A$, and note that $i q I J \subseteq A$ and $E_{i q}^{(I J)^{r}}(F)=F(i q)=i F(q)=$ $i E_{q}^{(I J)^{r}}(F)$ for all $F$ in $(I J)^{r}$. Therefore $E_{i q}^{(I J)^{r}}=\iota E_{q}^{(I J)^{r}}$. From this, taking into account the continuity of $\iota$ and of $E_{q}^{(I J)^{r}}$ (by Lemma 2), it follows that $E_{i q}^{(I J)^{r}}$ is bounded and $\left\|E_{i q}^{(I J)^{r}}\right\| \leq\|\iota\|\left\|E_{q}^{(I J)^{r}}\right\|$. Consequently, $i q$ lies in $Q_{b e}^{r}(A)$ and $\left|i q \mathbf{I}_{r} \leq\|\iota\|\right| \mathbf{|} q \mathbf{|}_{r}$. Thus, $Q_{b e}^{r}(A)$ is a complex subalgebra of $Q^{r}(A)$ and $\mathbf{I} \cdot \mathbf{I}_{r}$ is a real algebra norm on $Q_{b e}^{r}(A)$ which makes the multiplication by $i$ continuous. The proof is completed by applying [12; Theorem 1.3.3].

The central closure $Q(A)$ of a prime algebra $A$ is defined as the $C(A)$ subalgebra of $Q^{r}(A)$ generated by $A$. The central closure of a totally prime algebra may be normed in a suitable way as a consequence of Proposition 2 and Corollary 1(i).

Corollary 2. If $A$ is a totally prime real algebra with extended centroid $\mathbb{C}$, then there exists a complex algebra norm on $Q(A)$ such that the inclusions of $A$ into $Q(A)$ and of $M(A)$ into $M(Q(A))$ are topological and $Q(A)$ is a totally prime algebra.

Clearly, the reversal of sides allows us to state similar results for left algebras of quotients with bounded evaluation. The remainder of the paper is devoted to the study of symmetric algebras of quotients with bounded evaluation of totally prime algebras. We shall give a procedure that allows us to directly obtain the symmetric algebra of quotients with bounded eval- 
uation of a totally prime algebra, thus bypassing the one-sided algebras of quotients. For an ideal $I$ of an algebra $A$ we denote by $I^{m}$ the ideal of $M(A)$ generated by the set $\left\{M_{x, y}: x, y \in I\right\}$. It is clear that

$$
I^{m}=\left\{\sum_{i=1}^{n} M_{x_{i}, y_{i}}: n \in \mathbb{N}, x_{i}, y_{i} \in I(1 \leq i \leq n)\right\} .
$$

Furthermore, from the descriptions of $I^{r}, I^{l}$ and $I^{m}$, it follows that $I^{m} \subseteq$ $I^{r} \cap I^{l}$ and $I^{r} I^{l}=I^{l} I^{r}=I^{m}$.

Lemma 3. Let $A$ be a totally prime algebra, and assume that $K$ is a positive number such that

$$
K\|a\|\|b\| \leq\left\|N_{a, b}\right\|
$$

for all $a, b$ in $A$. Then, for $q$ in $Q^{r}(A)$ and a nonzero ideal $I$ of $A$ such that $q I \subseteq A$ we have:

$$
E_{q}^{I^{r}} \text { is bounded if, and only if, } E_{q}^{I^{m}} \text { is bounded, }
$$

and in that case

$$
K\left\|E_{q}^{I^{r}}\right\| \leq\left\|E_{q}^{I^{m}}\right\| \leq\left\|E_{q}^{I^{r}}\right\| .
$$

This statement remains valid if "right" is replaced by "left".

Proof. Let $q$ be in $Q^{r}(A)$ and $I$ be a nonzero ideal of $A$ such that $q I \subseteq A$. Since $I^{m} \subseteq I^{r}$, it follows that $E_{q}^{I^{m}}$ is bounded whenever $E_{q}^{I^{r}}$ is bounded, and in that case $\left\|E_{q}^{I^{m}}\right\| \leq\left\|E_{q}^{I^{r}}\right\|$. Suppose now that $E_{q}^{I^{m}}$ is bounded. For $x$ in $I, F$ in $I^{r}$ and $S, T$ in $M(A)$ we see that $L_{S(x)} T F$ belongs to $I^{m}$ and

$$
N_{x, F(q)}(S, T)=S(x) T F(q)=L_{S(x)} T F(q)=E_{q}^{I^{m}}\left(L_{S(x)} T F\right) .
$$

If additionally we assume that $\|x\|=1$ and $\|F\|=\|S\|=\|T\|=1$, it follows that

$$
\left\|N_{x, F(q)}(S, T)\right\|=\left\|E_{q}^{I^{m}}\left(L_{S(x)} T F\right)\right\| \leq\left\|E_{q}^{I^{m}}\right\|,
$$

and so $\left\|N_{x, F(q)}\right\| \leq\left\|E_{q}^{I^{m}}\right\|$. Since $A$ is totally prime we have $K\|F(q)\| \leq$ $\left\|E_{q}^{I^{m}}\right\|$, hence $K\left\|E_{q}^{I^{r}}(F)\right\| \leq\left\|E_{q}^{I^{m}}\right\|$. Therefore $E_{q}^{I^{r}}$ is bounded and $K\left\|E_{q}^{I^{r}}\right\|$ $\leq\left\|E_{q}^{I^{m}}\right\|$.

TheOREm 6. Let $A$ be a totally prime algebra, and assume that $K$ is a positive number such that

$$
K\|a\|\|b\| \leq\left\|N_{a, b}\right\|
$$

for all $a, b$ in $A$. Then

$$
\begin{array}{r}
Q_{b e}^{s}(A)=\left\{q \in Q^{s}(A): \text { there exists a nonzero ideal } I \text { of } A\right. \\
\text { such that } \left.q I+I q \subseteq A \text { and } E_{q}^{I^{m}} \text { is bounded }\right\},
\end{array}
$$


and $\mathbf{|} \cdot \mathbf{|}: Q_{b e}^{s}(A) \rightarrow \mathbb{R}$ defined by

$$
\mathbf{|} q \mathbf{|}=\inf \left\{\left\|E_{q}^{I^{m}}\right\|: I \text { is a nonzero ideal of } A\right.
$$

$$
\text { such that } \left.q I+I q \subseteq A \text { and } E_{q}^{I^{m}} \text { is bounded }\right\}
$$

is an algebra norm, which is equivalent to $\mid \cdot \mathbf{I}_{r}$ and to $\mathbf{|} \cdot \mathbf{I}_{l}$ (hence also to $\mathbf{I} \cdot \mathbf{I}_{s}$ ). Moreover:

(i) The inclusions of $A$ into $Q_{b e}^{s}(A)$ and of $M(A)$ into $M\left(Q_{b e}^{s}(A)\right)$ are topological. Precisely, $K^{2}\|a\| \leq|a| \leq\|a\|$ for all $a$ in $A$, and $K^{2}\|F\| \leq$ $|F| \leq\|F\|$ for all $F$ in $M(A)$.

(ii) $\left(Q_{b e}^{s}(A), \mathbf{I} \cdot \mathbf{I}\right)$ is a totally prime algebra. Precisely,

$$
K^{3}|p| \mathbf{|} q \mathbf{|} \leq \mathbf{|} N_{p, q}^{I^{m}} \times J^{m} \mathbf{I}
$$

for all $p, q$ in $Q_{b e}^{s}(A)$ and nonzero ideals $I, J$ of $A$ such that $p I+I p \subseteq A$ and $q J+J q \subseteq A$.

(iii) If $(Q, \rrbracket \cdot \rrbracket)$ is a normed algebra such that $Q$ is a subalgebra of $Q^{s}(A)$ containing $A$ and the inclusions of $A$ into $Q$ and of $M(A)$ into $M(Q)$ are topological, then $Q$ is contained in $Q_{b e}^{s}(A)$ and the inclusions of $Q$ into $Q_{b e}^{s}(A)$ and of $M(Q)$ into $M\left(Q_{b e}^{s}(A)\right)$ are continuous.

Proof. We will first prove the validity of the description of $Q_{b e}^{s}(A)$ given in the statement. If $q$ is in $Q_{b e}^{s}(A)$ and $I, J$ are nonzero ideals of $A$ such that $q I \subseteq A, J q \subseteq A$ and $E_{q}^{I^{r}}, E_{q}^{J^{l}}$ are bounded, then $I \cap J$ is a nonzero ideal of $A$ such that $q(I \cap J)+(I \cap J) q \subseteq A$ and, as a consequence of Lemma 3, $E_{q}^{(I \cap J)^{m}}$ is bounded and

$$
\left\|E_{q}^{(I \cap J)^{m}}\right\| \leq\left\|E_{q}^{I^{r}}\right\| \quad \text { and } \quad\left\|E_{q}^{(I \cap J)^{m}}\right\| \leq\left\|E_{q}^{J^{l}}\right\| .
$$

Conversely, suppose that for an element $q$ in $Q^{s}(A)$ there is a nonzero ideal $I$ of $A$ such that $q I+I q \subseteq A$ and $E_{q}^{I^{m}}$ is bounded. Then using Lemma 3 once again we see that $E_{q}^{I^{r}}, E_{q}^{I^{l}}$ are bounded and

$$
K\left\|E_{q}^{I^{r}}\right\| \leq\left\|E_{q}^{I^{m}}\right\| \quad \text { and } \quad K\left\|E_{q}^{I^{l}}\right\| \leq\left\|E_{q}^{I^{m}}\right\| .
$$

As a consequence, $q$ lies in $Q_{b e}^{r}(A) \cap Q_{b e}^{l}(A)=Q_{b e}^{s}(A)$.

Making minor changes in the proof of Theorem 1 we conclude that | $\mid$ is an algebra seminorm on $Q_{b e}^{s}(A)$ satisfying the inequalities $|a| \leq\|a\|$ for all $a$ in $A$ and $|F| \leq\|F\|$ for all $F$ in $M(A)$. Moreover, from (7) and (8) we deduce that $\mathbf{|} \cdot \mathbf{|}$ is equivalent to $\mid \cdot \mathbf{I}_{r}$ and to $\mid \cdot \mathbf{I}_{l}$. To be more precise,

$$
K \mathbf{|} \cdot \mathbf{I}_{r} \leq \mathbf{I} \cdot \mathbf{I} \leq \mathbf{|} \cdot \mathbf{I}_{r} \text { and } K \mathbf{|} \cdot \mathbf{I}_{l} \leq \mathbf{I} \cdot \mathbf{I} \leq \mathbf{I} \cdot \mathbf{I}_{l} .
$$

Now, by Theorem $5, K\|a\| \leq|a|_{r}$ for all $a$ in $A$, and therefore $K^{2}\|a\| \leq|a|$ for all $a$ in $A$ as a consequence of (9). From this, for each $F$ in $M(A)$ we have $K^{2}\|F(a)\| \leq|F(a)| \leq|F||a| \leq|F|\|a\|$ for all $a$ in $A$, and so 
$K^{2}\|F\| \leq \mathbf{| F |}$. At this point, making the necessary modifications in the proof of part (ii) of Theorem 5 we obtain part (ii) of our statement.

Finally, let $(Q,\|\cdot\|)$ be a normed algebra such that $Q$ is a subalgebra of $Q^{s}(A)$ containing $A$, and assume the existence of positive numbers $\alpha, \beta, \gamma, \delta$ such that $\alpha\|a\| \leq\|a \rrbracket \leq \beta\| a \|$ for all $a$ in $A$, and $\gamma\|F\| \leq\|F\| \leq \delta\|F\|$ for all $F$ in $M(A)$. Following the reasoning presented in the final part of the proof of Theorem 5, we see that $Q$ is continuously embedded in $Q_{b e}^{s}(A)$. Let $T$ be in $M(Q)$ and pick $n$ in $\mathbb{N}$ and $p_{1}, \ldots, p_{n}, q_{1}, \ldots, q_{n}$ in $Q^{1}$ (the unital envelope of $Q$ in $\left.Q^{s}(A)\right)$ such that $T=\sum_{i=1}^{n} M_{p_{i}, q_{i}}$. Given $q$ in $Q_{b e}^{s}(A)$, we can take a nonzero ideal $I$ of $A$ such that $q I+I q \subseteq A, E_{q}^{I^{m}}$ is bounded, and $p_{i} I+I p_{i} \subseteq A, q_{i} I+I q_{i} \subseteq A$ for all $i \in\{1, \ldots, n\}$. Since $T(q)=\sum_{i=1}^{n} p_{i} q q_{i}$, it is clear that $T(q) I^{3}+I^{3} T(q) \subseteq A$. Moreover, from the equality $M_{a, b} T=\sum_{i=1}^{n} M_{a p_{i}, q_{i} b}$, which holds for all $a, b$ in $A$, it follows that $\left(I^{3}\right)^{m} T \subseteq I^{m}$. Consequently, for every $F$ in $\left(I^{3}\right)^{m}$, we can write

$$
E_{T(q)}^{\left(I^{3}\right)^{m}}(F)=F T(q)=E_{q}^{I^{m}}(F T)
$$

and so

$$
\begin{aligned}
\left\|E_{T(q)}^{\left(I^{3}\right)^{m}}(F)\right\| & =\left\|E_{q}^{I^{m}}(F T)\right\| \leq\left\|E_{q}^{I^{m}}\right\|\|F T\| \leq \gamma^{-1}\left\|E_{q}^{I^{m}}\right\| \rrbracket F T \rrbracket \\
& \leq \gamma^{-1}\left\|E_{q}^{I^{m}}\right\| \rrbracket F \rrbracket \rrbracket T \rrbracket \leq \delta \gamma^{-1}\left\|E_{q}^{I^{m}}\right\|\|F\| \rrbracket T \rrbracket .
\end{aligned}
$$

Therefore, $E_{T(q)}^{\left(I^{3}\right)^{m}}$ is bounded and $\left\|E_{T(q)}^{\left(I^{3}\right)^{m}}\right\| \leq \delta \gamma^{-1}\left\|E_{q}^{I^{m}}\right\| \rrbracket T \rrbracket$. From this we deduce that $\left|T(q) \mathbf{|} \leq \delta \gamma^{-1}\right| q \mid \rrbracket T \rrbracket$, and as a consequence $|T| \leq \delta \gamma^{-1} \rrbracket T \rrbracket$.

Finally, we examine the symmetric algebra of quotients with bounded evaluation of totally multiplicatively prime algebras, which were recently introduced by the authors in [3]. These algebras are totally prime and may not be ultraprime. Recall that a normed algebra $A$ with nonzero product is totally multiplicatively prime (t.m.p. for short) if there exists a positive number $K$ such that $K\|F\|\|a\| \leq\left\|W_{F, a}\right\|$ for all $F$ in $M(A)$ and $a$ in $A$, where $W_{F, a}$ denotes the linear operator from $M(A)$ into $A$ defined by $W_{F, a}(T)=F T(a)$ for all $T$ in $M(A)$.

THEOREM 7. Let $A$ be a t.m.p. algebra, and assume that $K$ is a positive number such that

$$
K\|F\|\|a\| \leq\left\|W_{F, a}\right\|
$$

for all $F$ in $M(A)$ and $a$ in $A$. Then

(i) The inclusions of $A$ into $Q_{b e}^{s}(A)$ and of $M(A)$ into $M\left(Q_{b e}^{s}(A)\right)$ are topological. Precisely, $K\|a\| \leq|a| \leq\|a\|$ for all $a$ in $A$, and $K\|F\| \leq|F| \leq$ $\|F\|$ for all $F$ in $M(A)$.

(ii) $\left(Q_{b e}^{s}(A), \mathbf{|} \cdot \mathbf{I}\right)$ is a t.m.p. algebra. Precisely,

$$
K^{4}|F||q| \leq \mid W_{F, q}^{I^{m}} \mathbf{|}
$$


for all $F$ in $M\left(Q_{b e}^{s}(A)\right), q$ in $Q_{b e}^{s}(A)$ and nonzero ideals $I$ of $A$ such that $F I^{m}+I^{m} F \subseteq M(A)$ and $q I+I q \subseteq A$, where $W_{F, q}^{I^{m}}$ denotes the restriction of $W_{F, q}$ to $I^{m}$.

(iii) If $(Q, \rrbracket \cdot \rrbracket)$ is a normed algebra such that $Q$ is a subalgebra of $Q^{s}(A)$ containing $A$ and the inclusions of $A$ into $Q$ and of $M(A)$ into $M(Q)$ are topological, then $Q$ is contained in $Q_{b e}^{s}(A)$ and the inclusions of $Q$ into $Q_{b e}^{s}(A)$ and of $M(Q)$ into $M\left(Q_{b e}^{s}(A)\right)$ are continuous.

Proof. Taking Theorem 6 into account, all that remains to be proven is that $K\|a\| \leq|a|$ for all $a$ in $A, K\|F\| \leq|F|$ for all $F$ in $M(A)$, and that $\left(Q_{b e}^{s}(A), \mathbf{I} \cdot \mathbf{I}\right)$ is a t.m.p. algebra. Fix an element $a$ in $A$ and a nonzero ideal $I$ of $A$. For $F$ in $I^{m}$ and $G$ in $M(A)$ with $\|F\|=\|G\|=1$ we have

$$
\left\|W_{F, a}(G)\right\|=\|F G(a)\|=\left\|E_{a}^{I^{m}}(F G)\right\| \leq\left\|E_{a}^{I^{m}}\right\|,
$$

hence

$$
\left\|W_{F, a}\right\| \leq\left\|E_{a}^{I^{m}}\right\|
$$

and so $K\|a\| \leq\left\|E_{a}^{I^{m}}\right\|$. Taking the infimum over $I$ we conclude that $K\|a\|$ $\leq \mathbf{|} a \mathbf{|}$. Now, note that for $F$ in $M(A)$ we have

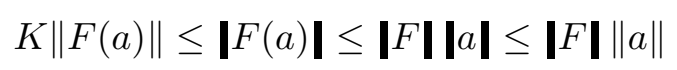

for all $a$ in $A$, and therefore $K\|F\| \leq|F|$.

To show that $\left(Q_{b e}^{s}(A), \mathbf{I} \cdot \mathbf{I}\right)$ is a t.m.p. algebra we first observe that if $F$ is in $M\left(Q_{b e}^{s}(A)\right)$ and if $I$ is a nonzero ideal of $A$ such that $F I^{m}+I^{m} F \subseteq A^{m}$, and if

$$
R_{F}^{I^{m}}:\left(I^{m},\|\cdot\|\right) \rightarrow\left(A^{m},\|\cdot\|\right)
$$

is the mapping defined by $R_{F}^{I^{m}}(T)=T F$ for all $T$ in $I^{m}$, then $R_{F}^{I^{m}}$ is bounded and

$$
K^{2}|F| \leq\left\|R_{F}^{I^{m}}\right\| \leq K^{-1}|F| \cdot
$$

Indeed, given $F$ in $M\left(Q_{b e}^{s}(A)\right)$ and a nonzero ideal $I$ of $A$ such that $F I^{m}+$ $I^{m} F \subseteq A^{m}$ we see that

$$
K\left\|R_{F}^{I^{m}}(T)\right\|=K\|T F\| \leq \mathbf{| T F |} \leq \mathbf{|} T \mathbf{|} F \mathbf{|} \leq\|T\| \mathbf{|} F \mathbf{|}
$$

for all $T$ in $I^{m}$, therefore $R_{F}^{I^{m}}$ is bounded and $\left\|R_{F}^{I^{m}}\right\| \leq K^{-1}|F|$. On the other hand, for each $q$ in $Q_{b e}^{s}(A)$ take a nonzero ideal $J$ of $A$ such that $F(q) J+J F(q) \subseteq A$, and note that for all $T$ in $(I \cap J)^{m}$ and $G$ in $M(A)$ with $\|T\|=\|G\|=1$ it follows that

$$
\begin{aligned}
K\left\|W_{\operatorname{Id}_{A}, T F(q)}(G)\right\| & =K\|G T F(q)\| \leq \mathbf{I} G T F(q) \mathbf{|} \leq \mathbf{|} G T F \mathbf{|} q \mathbf{|} \\
& \leq\|G T F\| \mathbf{|} q \mathbf{|}=\left\|R_{F}^{I^{m}}(G T)\right\| \mathbf{|} q \mathbf{|} \leq\left\|R_{F}^{I^{m}}\right\|\|G T\| \mathbf{|} q \mathbf{|} \\
& \leq\left\|R_{F}^{I^{m}}\right\| \mathbf{|} q \mathbf{\mathbf { I }},
\end{aligned}
$$


therefore $K\left\|W_{\operatorname{Id}_{A}, T F(q)}\right\| \leq\left\|R_{F}^{I^{m}}\right\| \mathbf{|} q \mathbf{|}$, and so $K^{2}\|T F(q)\| \leq\left\|R_{F}^{I^{m}}\right\||q|$. Rewriting this inequality in the form $K^{2}\left\|E_{F(q)}^{J^{m}}(T)\right\| \leq\left\|R_{F}^{I^{m}}\right\| \mathbf{|} q \mathbf{|}$, it follows that $K^{2}\left\|E_{F(q)}^{J^{m}}\right\| \leq\left\|R_{F}^{I^{m}}\right\| \mathbf{|} q \mathbf{|}$. Hence

$$
K^{2} \mid F(q) \mathbf{|} \leq\left\|R_{F}^{I^{m}}\right\| \mathbf{|} q \mathbf{|}
$$

and so $K^{2}|F| \leq\left\|R_{F}^{I^{m}}\right\|$.

Now, let $F$ be in $M\left(Q_{b e}^{s}(A)\right), q$ be in $Q_{b e}^{s}(A)$ and $I$ be a nonzero ideal of $A$ such that $F I^{m}+I^{m} F \subseteq M(A)$ and $q I+I q \subseteq A$. For $G, H$ in $I^{m}$ and $T$ in $M(A)$ with $\|G\|=\|H\|=\|T\|=1$ we have

$$
\begin{aligned}
& K\left\|W_{G F, H(q)}(T)\right\|=K\|G F T H(q)\| \leq K\|F T H(q)\| \leq \mathbf{| F T H ( q ) |} \\
& =\left|W_{F, q}^{I^{m}}(T H) \mathbf{|} \leq \mathbf{|} W_{F, q}^{I^{m}} \mathbf{|}\right| \mathbf{T H}|\leq| W_{F, q}^{I^{m}} \mathbf{|}\|T H\| \\
& \leq \mathbf{|} W_{F, q}^{I^{m}} \mathbf{|},
\end{aligned}
$$

and therefore $K\left\|W_{G F, H(q)}\right\| \leq \mid W_{F, q}^{I^{m}} \mathbf{I}$. Since $A$ is t.m.p. it follows that $K^{2}\|G F\|\|H(q)\| \leq \mathbf{|} W_{F, q}^{I^{m}} \mathbf{|}$. Rewriting this inequality in the form

$$
K^{2}\left\|R_{F}^{I^{m}}(G)\right\|\left\|E_{q}^{I^{m}}(H)\right\| \leq \mid W_{F, q}^{I^{m}} \mathbf{I}
$$

we can deduce that

$$
K^{2}\left\|R_{F}^{I^{m}}\right\|\left\|E_{q}^{I^{m}}\right\| \leq \mid W_{F, q}^{I^{m}} \mathbf{|} .
$$

Now, applying our previous statement, we obtain $K^{4}|F|\left\|E_{q}^{I^{m}}\right\| \leq \mid W_{F, q}^{I^{m}} \mathbf{I}$, and as a consequence

$$
K^{4}|F||q| \leq \mid W_{F, q}^{I^{m}} \mathbf{|}
$$

\section{References}

[1] S. A. Amitsur, On rings of quotients, Symposia Math. 8 (1972), 149-164.

[2] K. I. Beidar, W. S. Martindale III, and A. V. Mikhalev, Rings with Generalized Identities, Dekker, New York, 1996.

[3] M. Cabrera and A. A. Mohammed, Totally multiplicatively prime algebras, Proc. Roy. Soc. Edinburgh Sect. A, to appear.

[4] M. Cabrera and A. Rodríguez, Nonassociative ultraprime normed algebras, Quart. J. Math. Oxford (2) 43 (1992), 1-7.

[5] W. S. Martindale III, Prime rings satisfying a generalized polynomial identity, J. Algebra 12 (1969), 576-584.

[6] M. Mathieu, Applications of ultraprime Banach algebras in the theory of elementary operators, thesis, Tübingen, 1986.

[7] - Rings of quotients of ultraprime Banach algebras with applications to elementary operators, Proc. Centre Math. Anal. Austral. Nat. Univ. 21 (1989), 297-317.

[8] - The symmetric algebra of quotients of an ultraprime Banach algebra, J. Austral. Math. Soc. Ser. A 50 (1991), 75-87.

[9] D. S. Passman, Computing the symmetric ring of quotients, J. Algebra 105 (1987), 207-235. 
[10] G. K. Pedersen, Analysis Now, Springer, New York, 1988.

[11] A. Pietsch, Operator Ideals, North-Holland, Amsterdam, 1980.

[12] C. E. Rickart, General Theory of Banach Algebras, Krieger, New York, 1960.

[13] J. R. Ringrose, Compact Non-Self-Adjoint Operators, Van Nostrand Reinhold, London, 1971.

[14] R. Schatten, Norm Ideals of Completely Continuous Operators, Springer, New York, 1970 .

Departamento de Análisis Matemático

Department of Mathematics

Facultad de Ciencias

College of Education

Universidad de Granada

University of Mosul

18071 Granada, Spain

Mosul, Iraq

E-mail: cabrera@goliat.ugr.es

Received February 12, 2001

Revised version June 3, 2002 